\title{
Immunohistochemistry and alternative FISH testing in breast cancer with $H E R 2$ equivocal amplification
}

\author{
Sally Agersborg ${ }^{1} \cdot$ Christopher Mixon $^{1} \cdot$ Thanh Nguyen $^{1} \cdot$ Sramila Aithal $^{2} \cdot$ Sucha Sudarsanam $^{1} \cdot$ Forrest Blocker $^{1}$. \\ Lawrence Weiss $^{1} \cdot$ Robert Gasparini $^{1}$. Shiping Jiang ${ }^{1} \cdot$ Wayne Chen $^{1} \cdot$ Gregory Hess $^{3} \cdot$ Maher Albitar $^{1}$ (i)
}

Received: 15 November 2017 / Accepted: 13 March 2018 / Published online: 22 March 2018

(c) The Author(s) 2018

\begin{abstract}
Purpose While HER2 testing is well established in directing appropriate treatment for breast cancer, a small percentage of cases show equivocal results by immunohistochemistry (IHC) and fluorescence in situ hybridization (FISH). Alternative probes may be used in equivocal cases. We present a single community-based institution's experience in further evaluating these cases.

Patients and methods Between 2014 and 2016, 4255 samples were submitted for HER2 amplification testing by alternative probes, TP53, RAII, and RARA. Of the patients tested by FISH, 505/3908 (12.9\%) also had IHC data.

Results Most (73.9\%) FISH equivocal cases remained equivocal after IHC testing. However, 50.5\% of equivocal cases were classified as HER 2 amplified by alternative probes. Most cases were positive by more than one probe: $78 \%$ of positive cases by $R A I I$ and $73.9 \%$ by TP53. There was a significant difference between IHC and FISH alternative testing $(p<0.0001)$ among the equivocal cases by conventional FISH testing, $44 \%$ of IHC negative cases became positive while $36 \%$ of the positive IHC cases became negative by alternative FISH testing. Available data showed that $41 \%$ of patients were treated with palbociclib and were positive by alternative FISH.

Conclusion The prevalence of double HER2 equivocal cases and the discrepancy between IHC and alternative FISH testing suggest that FISH alternative testing using both RAII and TP53 probes is necessary for conclusive classification. Because almost half of FISH equivocal cases converted to HER 2 amplified upon alternative testing, clinical studies to determine the benefit of anti-HER2 therapy in these patients are urgently needed.
\end{abstract}

Keywords HER2 $\cdot$ Equivocal $\cdot$ FISH $\cdot$ IHC $\cdot$ Breast cancer

\section{Introduction}

Testing human epidermal growth factor receptor 2 gene amplification (HER2; ERBB2) is important in breast cancer (BC) and other cancers. HER2, on the long arm of chromosome 17, is amplified and its encoded protein is overexpressed in $10-25 \%$ of BCs [1]. Without anti-HER2 treatment, HER2-amplified tumors are associated with poor

Maher Albitar

maheralbitar@gmail.com

1 NeoGenomics Laboratories, Research and Development, 31 Columbia, Aliso Viejo, CA 92656, USA

2 Cancer Treatment Centers of America, Philadelphia, PA, USA

3 Symphony Health, Conshohocken, PA, USA prognosis [1,2], but when treated with therapies targeting HER2, such as trastuzumab, pertuzumab, and TDM-1, they are shown to markedly improve survival [3-5]. Moreover, these patients can be excluded from treatment for which HER2-amplified tumors have been shown to be resistant, such as anthracyclines, taxanes, cyclophosphamides, and tamoxifen [6]. Anti-HER2 therapy is expensive and cardiotoxic in some patients, and HER2-unamplified patients should not be prescribed HER2 inhibitors [4, 7, 8].

HER 2 amplification testing determines if a patient is eligible to be treated with anti-HER2 therapy [9-11]. HER2 amplification testing is performed either using immunohistochemical analysis (IHC), which tests for protein expression, or fluorescence in situ hybridization (FISH), which tests for gene amplification [2]. Many laboratories use IHC analysis as a primary assay, with "reflex" FISH testing for IHC equivocal results. In 2013, the American Society of 
Clinical Oncology and the College of American Pathologists (ASCO-CAP) updated the HER2 interpretative guidelines and revised the equivocal category for HER 2 ISH, proposing a reflex test for such cases on the same specimen, with a different method or with an alternate ISH chromosome probe [12]. Based on the 2013 ASCO-CAP guidelines, a sample is classified as equivocal by FISH when HER2:CEP17 (centromere) is $<2.0$, but HER2 signals are $\geq 4$ but $<6$ [12]. The current ASCO-CAP guidelines increased the number of equivocal cases significantly from the previous 2007 one, so that $10-17 \%$ of tested BCs are estimated to be classified as equivocal by the new ASCO-CAP FISH testing guidelines [13-20].

A probe at or near centromere 17 has traditionally been used as a reference probe, because it presumably reflects the copy number of chromosome 17 [21]. However, the centromere probe region can also be amplified as a result of local or regional amplification [22-25]. Moreover, HER2amplified cancers have complex genetic abnormalities and the HER2 amplicon at 17q12 contains multiple genes, which may co-amplify with HER2 [26]. The use of alternative chromosome 17 probes, including $R A I I$ (retinoic acid induced 1; previously known as $S M S$ ), RARA (retinoic acid receptor), and TP53 (tumor protein 53), allows an alternative calculation of the HER2:chromosome 17 ratio, providing an alternative FISH ratio score in cases with a complex $C E P 17$ pattern, including polysomy 17 [12, 21, 25]. The 2013 ASCO-CAP guidelines do not specify which alternative probes should be used or whether guidelines for FDA-approved probes can be extrapolated to alternate probes [12]. Alternative chromosome 17 probes currently used in these cases are RARA, TP53, and RAII. These loci are distal from HER2 on the long and short arms of chromosome 17 and in principle are presumed to be less influenced by HER 2 amplification [21]. In this paper, we report one reference laboratory's experience in testing HER2 equivocal cases referred from various institutions.

\section{Methods}

\section{Patient samples and methodology}

Between late 2014 and late 2016, 4255 consecutive formalin-fixed paraffin-embedded (FFPE) BC samples were submitted for testing at NeoGenomics Laboratories (Aliso Viejo, CA) by alternative probes for HER2 amplification due to equivocal results by conventional methods. The list of patients tested with alternative $H E R 2$ probes was processed to generate HIPAA-compliant synthetic identifiers, subsequently submitted to Symphony Health (Conshohocken, PA) to obtain clinical information, including drug exposure [27]. By utilizing the same de-identification algorithm at
Symphony, all patient-level records were matched and linked across setters of care. Symphony Health Solutions' data warehouse contains longitudinal patient (220 million) data sources that capture adjudicated prescription (10 billion), medical, and hospital claims across the United States. This study was approved by the Western Institutional Review Board, Olympia, WA.

\section{Immunohistochemistry testing}

From each tissue block, $4 \mu \mathrm{m}$ sections were cut, deparaffinized in xylene, and dehydrated through alcohol changes. IHC for HER2 was performed with one of the FDA-approved assay kits (Lica Biosystem), according to standard methods as previously published and scored according to the 2013 ASCO-CAP Guidelines [28].

\section{Fluorescence in situ hybridization testing}

Three probe kits were used for FISH analysis: (1) the US Food and Drug Administration (FDA)-approved HER2 IQFISH pharmDx (Agilent, Santa Clara, California). HER2 equivocal panel contained RARA (17q21.2), RAII (17p11.2), TP53 (17p13.1), and CEP17 (SHGC133091-RH42746) (Agilent, Santa Clara, California) for the HER2 alternative panel. This $C E P 17$ probe is $436 \mathrm{kB}$, paracentromeric, and located at $17 \mathrm{p} 11.2$, while the centromeric probe used in conventional HER2 testing (CEN17) is located between 17p11.1-q11.1.

For each specimen, $4 \mu \mathrm{m}$ sections were cut, deparaffinized, rehydrated, and heated in pretreatment solution [2-(N-morpholino) ethanesulphonic acid] at $97{ }^{\circ} \mathrm{C}$ for $10 \mathrm{~m}$ and then soaked in Tris/ $\mathrm{HCl}$ buffer at room temperature (RT) for $3 \mathrm{~m}$, twice. Then, the specimens were digested by proteinase $\mathrm{K}(25 \mathrm{mg} / \mathrm{mL})$ at $37^{\circ} \mathrm{C}$ for $45 \mathrm{~m}$ and agitated in Tris/ $\mathrm{HCl}$ buffer at RT for $3 \mathrm{~m}$, twice. Slides were then dehydrated in ethanol series at RT and air-dried. Ten $\mu$ of probe was applied to each slide, and the hybridization area was coverslipped. Co-denaturation of probe and specimen was performed at $66{ }^{\circ} \mathrm{C}$ for $10 \mathrm{~m}$, and the slides were incubated at $45{ }^{\circ} \mathrm{C}$ for $90 \mathrm{~m}$. Slides were washed in $2 \times \mathrm{SSC} / 0.3 \% \mathrm{NP}-40$ at $63{ }^{\circ} \mathrm{C}$ for $10 \mathrm{~m}$, and $10 \mu \mathrm{l}$ of DAPI ( $4^{\prime} 6^{\prime}$ - diamidino-2-phenylindole dihydrochloride) counterstain was applied before coverslipping. Slides were analyzed on a Zeiss Imager. Z2 fluorescent microscope using Isis FISH imaging systems (MetaSystems, Altlussheim, Germany) software. For each slide, fluorescent signals were counted by two independent observers in 40 cells (ASCO-CAP guidelines recommend a minimum of 20 cells per slide) [28]. Equivocal status for $H E R 2$ was determined by the ratio of HER2:CEP $17<2$ with an average $H E R 2$ copy number $\geq 4.0$ but $<6.0$. Alternative testing was considered positive if the ratio using one of the alternative probes was $\geq 2.0$. TP53 scoring was not 
considered when the TP53 signals were $<1.7$ because the low number of signals in these cases is consistent with TP53 $17 \mathrm{p}$ deletion rendering the probe an invalid control for ratio determination in these cases. In addition, a normalization approach was used as follows: HER 2 x CEP17/TP53 3 CEP17.

\section{Statistical analyses}

Counts and frequencies were reported for each pre-specified measure and endpoint. The level of significance was defined as $\alpha=0.05$. Standard statistical tests were used to evaluate the correlations between variables including the Wilcoxon Matched Pairs Test.

\section{Results}

\section{Patient characteristics}

Of the tested 4255 FISH equivocal samples, 282 were duplicate samples either due to an inadequate first sample or due to testing of a second paraffin block from the same tumor. Therefore, there were 3973 unique patient samples. Of these, 65 had either inadequate tumor for evaluation (QNS) or inadequate number cells counted for conclusive results, yielding 3908 unique samples with FISH data (Table 1). Of the 3973 total unique patient samples, $97.0 \%(N=3854)$ were matched to one or more claims data. The patient demographic profile of the study is 58\% Caucasian, $10 \%$ African American, 4\% Hispanic, and other or missing data in $28 \%$.

\section{IHC in HER2 FISH equivocal cases}

Of the 3908 patients tested by FISH, 556 (14.2\%) also had IHC data. These data are summarized in Table 1. Of the 556 FISH equivocal cases, IHC was negative $($ score $=0 / 1+)$ in $137 / 556$ (24.6\%), and positive (score $=3+$ ) in 13/556 (2.3\%), and the rest, $406 / 556$ (73.9\%), were equivocal (double equivocal) by conventional testing.

Table 1 FFPE samples available for IHC and FISH alternative testing

\begin{tabular}{lcllcr}
\hline \multicolumn{2}{l}{ FISH alternative testing } & & \multicolumn{3}{l}{ IHC (all cases) } \\
\cline { 1 - 2 } \cline { 5 - 6 } FISH result & Cases $(N)$ & & IHC score & Cases $(N)$ & $\%$ \\
\hline Duplicate & 282 & & & \\
QNS & 65 & & $0 / 1$ & 137 & 24.6 \\
Positive & 1973 & & 3 & 13 & 2.3 \\
Negative & 1935 & & 2 & 406 & 73.0 \\
Total & 4255 & & 556 & 100.0 \\
\hline
\end{tabular}

$Q N S$ inadequate tumor for evaluation

\section{Duplicate testing by IHC}

Patients tested by IHC included 96 cases tested twice using a different paraffin block from the same tumor. In the first test, 4/96 (4.2\%) demonstrated an IHC score of 0, 32/96 $(33.3 \%)$ a score of $1+, 57 / 96(59.4 \%)$ a score of $2+$, and $3 / 96(3.1 \%)$ a score of $3+$. In the second test, $6 / 96(6.3 \%)$ scored 0, 39/96 (40.6\%) scored 1+, 47/96 (49.0\%) scored $2+$, and $4 / 96(4.2 \%)$ scored 3 . These results are shown in Table 2. There was a significant difference $(p=0.001)$ in the groups classified as $2+$ and 1+by first IHC: $44 \%$ of cases scored as $1+$ on the first IHC test became equivocal (score $2+$ ) on the second block test, and $40 \%$ of the equivocal cases on the first block test scored as negative and $7 \%$ as positive on the second block test (Table 2).

\section{Discrepancy between IHC and alternative fish testing}

After duplicates were excluded, 507 FISH equivocal cases had complete IHC and alterative FISH testing. As shown in Table 3, of the 121 negative cases by IHC (score 0 and $1+), 43.8 \%$ (53/121) became positive by alternative FISH. Although the number is small, $36.4 \%$ (4/11) of the positive cases by IHC became negative by alterative FISH testing. Of the equivocal cases by IHC (score $3+$ ), $52.3 \%$ became positive. This was statistically significant $(p<0.0001)$. The high discordance in these cases as compared with average $\mathrm{BC}$ cases, most likely, reflects that the HER2 equivocal cases by FISH are different with borderline amplification.

\section{Duplicate testing by FISH in HER2 equivocal cases}

Of the cases tested by alternative FISH testing, 200 were tested twice using a different paraffin block. There was a significant difference in results between the first and the

Table 2 Duplicate testing by IHC using a different paraffin block of the same tumor

\begin{tabular}{lllrrl}
\hline $\begin{array}{l}\text { First IHC } \\
\text { score }\end{array}$ & No & $\begin{array}{l}\text { Second IHC } \\
\text { score }\end{array}$ & No & $\%$ & $p$ value \\
\hline 0 & 4 & 0 & 1 & 25.0 & NA \\
& & 1 & 3 & 75.0 & \\
1 & 32 & 0 & 2 & 6.3 & $p=0.001$ \\
& & 1 & 16 & 50.0 & \\
& & 2 & 14 & 43.8 & \\
2 & 57 & 0 & 3 & 5.3 & $p=0.001$ \\
& & 1 & 20 & 35.1 & \\
& & 2 & 30 & 52.6 & \\
3 & & 3 & 4 & 7.0 & \\
& 3 & 2 & 3 & 100.0 & NA \\
\hline
\end{tabular}


Table 3 Discrepancy between IHC and alternative FISH testing

\begin{tabular}{|c|c|c|c|c|c|c|c|c|}
\hline \multirow[t]{2}{*}{ Alternative FISH } & \multicolumn{2}{|c|}{$\begin{array}{l}\text { IHC score } 0 \\
\text { Negative }\end{array}$} & \multicolumn{2}{|c|}{$\begin{array}{l}\text { IHC score } 1 \\
\text { Negative }\end{array}$} & \multicolumn{2}{|c|}{$\begin{array}{l}\text { IHC score } 2 \\
\text { Equivocal }\end{array}$} & \multicolumn{2}{|c|}{$\begin{array}{l}\text { IHC score } 3 \\
\text { Positive }\end{array}$} \\
\hline & No & $\%$ & No & $\%$ & No & $\%$ & No & $\%$ \\
\hline Negative & 14 & 63.6 & 54 & 54.5 & 178 & 47.7 & 4 & 36.4 \\
\hline Positive & 8 & 36.4 & 45 & 45.5 & 195 & 52.3 & 7 & 63.6 \\
\hline Total & 22 & 100.0 & 99 & 100.0 & 373 & 100.0 & 11 & 100.0 \\
\hline
\end{tabular}

Table 4 Comparison between probes results in duplicate testing

\begin{tabular}{lllc}
\hline Pair of variables & & $Z$ & $p$ value \\
\cline { 1 - 2 } Measured probe & Reference probe & & \\
\hline RAI1 & TP53 & 2.01977 & 0.04 \\
$R A I 1$ & RARA & 6.87694 & $<0.00001$ \\
$R A I 1$ & RAI1 & 0.53653 & 0.59 \\
$R A R A$ & TP53 & 8.168 & $<0.00001$ \\
$R A R A$ & RARA & 0.09232 & 0.93 \\
TP53 & TP53 & 0.49738 & 0.62 \\
TP53 & RARA & 8.27438 & $<0.00001$ \\
$C E P 17$ & $C E P 17$ & 0.57489 & 0.57 \\
HER2 & HER2 & 0.73485 & 0.46 \\
$C E P 17$ & $D 17 Z 1$ & 5.59557 & $<0.00001$ \\
\hline
\end{tabular}

second test $(p<0.00001)$ when cases with complete results are considered. Some of the repeat testing was due to inadequate tumor for evaluation or indeterminate results due to availability of less than 50 cells for counting. If these cases are excluded, 18 of 97 negative cases $(18.6 \%)$ became positive on second block testing and 16 of 82 of positive cases (19.5\%) became negative on second block testing. The reason for this difference does not seem to be related to significant differences in the results of the same probes used in the first block test as compared with the second block test (Table 4). There was significant difference in results between different probes as well as between the two control probes used in the HER2 testing: $C E N 17$ and the paracentromeric probe used with the TP53 testing (CEP17). The difference in positive versus negative results is most likely due to minor difference in signals that may reflect heterogeneity within the tumor.

\section{Alternative testing results}

After excluding duplicates and cases without complete results, 1973 cases $(50.5 \%$ )were reported positive by alternative HER2 FISH testing, and 1935 cases (49.5\%) were reported as negative (see Table 1 ). There was no significant difference in age $(p=0.06)$ between the two groups. However, there was significant $(p<0.0001)$ difference in the scores between the three probes (RAIl, TP53, and RARA). As shown in Fig. 1, thirty-eight percent of all cases (78\% of positive cases) were deemed positive based on the RAII probe, $36 \%$ of all cases ( $73 \%$ of positive cases) were considered positive based on the TP53 probe, but only $30 \%$ of these cases were uniquely positive in TP53 (11\% of all cases and $22 \%$ of positive cases). This agrees with a previous study showing that $R A I$-identified FISH equivocal cases as amplified in almost $40 \%$ of all cases when used as the only alternative probe [12]. Only $5 \%$ of all cases ( $9 \%$ of positive cases) were considered positive based on RARA. However, if we exclude cases with $R A I I$ signals $<1.7$, in a fashion similar to our approach to TP53 probe, $10 \%$ of positive cases by $R A I I$

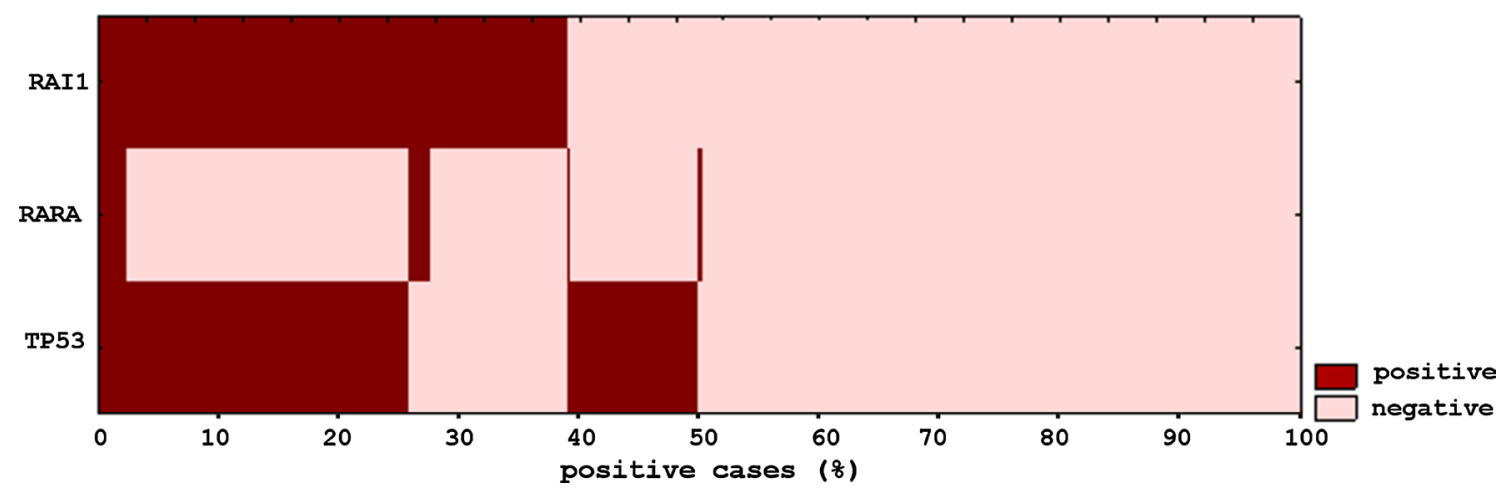

Fig. 1 Representation of the overlap in results of the three probes used in classifying the tested equivocal cases. $38 \%$ of all cases were deemed positive based on RAII probe, $36 \%$ based on the TP53 probe, and 5\% based on RARA 
would be excluded, but half, $5 \%$, of these cases would have been considered amplified by TP53 probe.

Furthermore, it has been suggested that to account for variations in signal counts between tissue sections, the HER2:TP53 ratio can be normalized based on the centromere counts in the two tissue sections and can be calculated as

\section{$\frac{H E R 2 x[C E P 17 \text { ofTP53] }}{\text { TP53x[CEN17ofHER2] }}$.}

18.5 of total cases were positive by normalization (excluding any case with TP53 signal $<1.7$ ). Using this approach increases the positive cases to $54.2 \%$ of all equivocal cases. However, only $6 \%$ of the positive cases would be called positive based solely on normalization. Since there is a significant difference between the two centromere probes used, we do not think this normalization is appropriate and these cases are not reported as positive for HER 2 amplification in this study.

\section{Estrogen/progesterone (ER/PR) receptors status in HER2 equivocal cases}

ER/PR evaluation using IHC was available on 225 samples that were classified as equivocal for HER 2 by conventional testing. Of these $80(35.5 \%)$ were reclassified as positive, $86(38.2 \%)$ were reclassified as negative, and $59(26.2 \%)$ remained as equivocal after alternative testing (Table 5). Significantly, more positive cases for HER 2 by alternative testing were ER+/PR+ as compared with HER2 negative by alternative testing: $82 \%$ versus $59 \%(p=0.0009)$. This is consistent with the expected higher hormone receptors positivity in cases with HER 2 positivity and supports that alternative HER2 testing provides data in line with the conventional testing [29]. In contrast, cases remained equivocal for HER2 testing by alternative testing, which showed no significant difference in ER/PR positivity from the HER2 positive, $75 \%$ versus $82 \%(p=0.15)$ or negative, $75 \%$ versus $59 \%(p=0.14)$.

\section{Therapy data in patients with equivocal fish testing}

Although data may not complete, based on the information available in the claims data, few of these patients $(N=16)$ were treated with $H E R 2$ inhibitors, while 124 patients were treated with palbociclib therapy, which is recommended for HER2-negative tumors. Therefore, proportionally few patients were treated according to the results of the alternative FISH testing, despite the availability of the results of the testing. However, this may not be accurate because of the pattern of dispensing anti-HER2 therapy in a hospitalbased setting and some of these data may be absent from the dataset. Nevertheless, of the 16 patients treated with antiHER2 therapy, two were negative by alternative FISH testing and 14 were positive. Pertuzumab was used in six of the trastuzumab-treated patients, five of whom were HER 2 positive by alternative testing. Of the 124 patients treated with palbociclib, $51(41 \%)$ were positive for HER2 by alternative FISH testing. To explore if there was an overall difference in the prescribed medications between cases classified as HER 2 positive and negative by alternative FISH testing, we grouped the therapy into the following categories: aromatase inhibitors, antineoplastic anti-estrogens, menopause-estrogen alone, pyrimidine analogs, serine-threonine kinase inhibitors, antineoplastic progestins, antineoplastic monoclonal antibodies, tyrosine kinase inhibitors, nitrogen mustards, and taxoids. There was no significant difference in therapy when drugs were grouped into these classes between positive and negative $H E R 2$ cases by alternative testing (Fig. 2). There were 34 patients treated with everolimus. Of these, 14 were classified as HER2 unamplified and 20 as HER2 amplified.

\section{Discussion}

While the overall concordance between FISH and IHC is high (94.1-97.6\% in average BC cases), and a definitive result by IHC will be largely consistent with FISH, equivocal cases differ substantially [30-33]. Studies have shown that 20-28\% IHC equivocal cases have gene amplification by FISH [20, 28, 30, 34]. The 2013 ASCO-CAP criteria for determining HER 2 amplification by FISH increase the number of equivocal cases significantly from the earlier 2007 criteria [21]. Classifying and understanding the biological and clinical behavior of equivocal cases is more difficult and requires more carefully designed biomarkers. Alternative FISH probes have been recommended for equivocal cases, but the reliability of such testing and the clinical relevance of the results are not well established. In the current study, we attempted to explore the relationship between IHC and
Table 5 Correlation between HER2 and HR status

\begin{tabular}{llllllll}
\hline & No & ER+ & \multicolumn{3}{c}{ PR+ } & \multicolumn{2}{c}{ ER +/PR+ } \\
\hline HER2+ & 80 & 71 & $88.8 \%$ & 66 & $82.5 \%$ & 66 & $82.5 \%$ \\
HER2- & 86 & 62 & $72.1 \%$ & 54 & $62.8 \%$ & 51 & $59.3 \%$ \\
HER2 equivocal & 59 & 52 & $88.1 \%$ & 46 & $78.0 \%$ & 44 & $74.6 \%$ \\
\hline
\end{tabular}




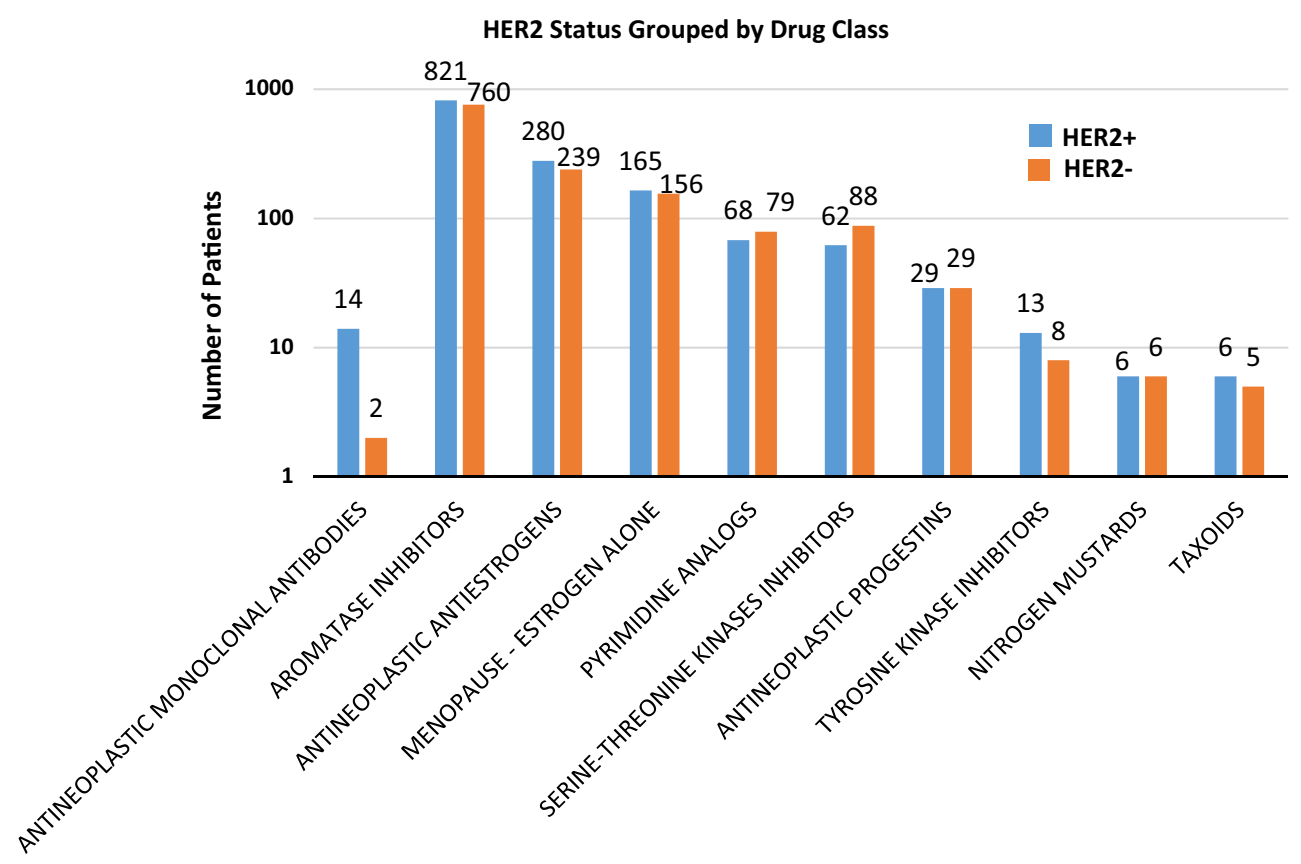

Fig. 2 Except for treatment with anti-HER2, there was no significant difference in overall therapy between HER2-positive and HER2-negative cases as assessed by alternative FISH testing. Drugs are grouped into classes as shown

FISH alternative testing in a large number of communitybased samples that were referred to one reference laboratory and tested in relatively similar fashion. We also attempted to explore trends for treating this group of patients in community-based oncology practice.

Based on our data, $73.9 \%$ of FISH equivocal cases remained equivocal after IHC testing. Therefore, relying on IHC for resolving FISH equivocal cases is not adequate and most cases need to be resolved by other means. Furthermore, the poor reproducibility of IHC in HER2 testing [35, 36] and the significant discrepancy observed upon repeated testing of samples on a different block, in the same laboratory, using the same conditions, and interpreted by the same pathologists, confirm that relying on IHC in these cases may not be an acceptable approach. Our alternative FISH testing resulted in $52 \%$ of the equivocal cases becoming positive for HER2 amplification. Furthermore, there was significant $(p<0.0001)$ difference between IHC testing and FISH alternative testing. This discrepancy most likely reflects that equivocal cases are different from average $\mathrm{BC}$ cases in terms of detecting HER 2 amplification. In fact, the difference in results when different blocks were tested by alternative FISH probes also suggests that these cases are different from average cases. In general, FISH testing is more reproducible and objective. The reproducibility seen in duplicate IHC testing is significantly lower ( $44 \%$ of IHC negative became equivocal and $40 \%$ of IHC equivocal became negative) than FISH (19\% of alternative FISH positive cases became negative and $20 \%$ became positive on duplicate alternative FISH testing).
There was a significant $(p<0.0001)$ difference in the scores obtained by each of the three probes (RAII, TP53, and RARA), suggesting that they are not redundant. The $R A I I$ probe was used for final classification of $78 \%$ of the positive cases. The TP53 probe as a single marker for amplification was used in only $22 \%$ of cases. RARA was the single marker for amplification in only $5 \%$ of positive cases, which suggests that it has limited discriminatory value.

Clearly, FISH alternative testing provides valuable information and is relatively more precise than IHC in classifying HER2 amplification. The demonstration that there is a significantly higher percentage of patients positive for ER+/ $\mathrm{PR}+$ in the cases that converted to HER 2 positive by alternative testing also supports the value of alternative testing by FISH. Although a large percentage of equivocal cases become amplified, making them eligible for HER2-targeted therapies, there are no data available to determine whether this subgroup of patients will benefit. There is a clear necessity to evaluate response in patients identified as amplified by alternate probe in a clinical trial such as NCT01275677. In principle, approximately half of the patients in this study should have been considered for targeted anti-HER2 therapy. Although accurate and complete data on therapy in these community-based patients are not available, based on the available information, few of these eligible patients $(N=15)$ were treated with HER 2 inhibitors. In contrast, $41 \%$ of patients who received a therapy that is indicated in HER2negative patients were $H E R 2$ positive by alternative FISH testing. Despite the concern over incompleteness of the data, 
the difference in number of patients treated with anti-HER2 therapy versus those who were treated as if $H E R 2$ was not amplified, which suggests that in community-based practice, a high percentage of patients with HER2 amplification are not being treated with HER2 inhibitors.

\section{Conclusion}

In this study, we demonstrated that the majority of BCs with equivocal HER2 FISH results remain equivocal after IHC testing. Alternative FISH testing of HER2 amplification in equivocal cases provides a standardized approach resulting in classifying almost half the cases as amplified. While currently there are no conclusive data to support that these patients will respond to anti-HER2 therapy and clinical trials are needed to specifically answer this question, patients classified as HER2 equivocal should be tested by alternative probes and should be considered for anti-HER2 therapy when positive.

\section{Compliance with ethical standards}

Conflict of interest Sally Agersborg, Christopher Mixon, Thanh Nguyen, Sucha Sudarsanam, Forrest Blocker, Lawrence Weiss, Bob Gasparini, Shiping Jiang, Wayne Chen, and Maher Albitar are employed by NeoGenomics, an oncology reference laboratory. Gregory Hess is employed by Symphony Health, a medical data analytics company.

Open Access This article is distributed under the terms of the Creative Commons Attribution 4.0 International License (http://creativeco mmons.org/licenses/by/4.0/), which permits unrestricted use, distribution, and reproduction in any medium, provided you give appropriate credit to the original author(s) and the source, provide a link to the Creative Commons license, and indicate if changes were made.

\section{References}

1. Emde A, Köstler WJ, Yarden Y (2012) Therapeutic strategies and mechanisms of tumorigenesis of HER2-overexpressing breast cancer. Crit Rev Oncol Hematol 84:e49-e57

2. Hilal T, Romond EH (2016) ERBB2 (HER2) testing in breast cancer. JAMA 315(12):1280-1281

3. Vogel CL, Cobleigh MA, Tripathy D et al (2002) Efficacy and safety of trastuzumab as a single agent in first-line treatment of HER2-overexpressing metastatic breast cancer. J Clin Oncol 20(3):719-726

4. Piccart-Gebhart MJ, Procter M, Leyland-Jones B et al (2005) Trastuzumab after adjuvant chemotherapy in HER2-positive breast cancer. N Engl J Med 353(16):1659-1672

5. Kaufman B, Trudeau M, Awada A et al (2009) Lapatinib monotherapy in patients with HER2-overexpressing relapsed or refractory inflammatory breast cancer: final results and survival of the expanded HER2 + cohort in EGF103009, a phase II study. Lancet Oncol. 10(6):581-588
6. Ménard S, Valagussa P, Pilotti S et al (2001) Response to cyclophosphamide, methotrexate, and fluorouracil in lymph node-positive breast cancer according to HER2 overexpression and other tumor biologic variables. J Clin Oncol 19(2):329-335

7. Cobleigh MA, Vogel CL, Tripathy D et al (1999) Multinational study of the efficacy and safety of humanized anti-HER2 monoclonal antibody in women who have HER2-overexpressing metastatic breast cancer that has progressed after chemotherapy for metastatic disease. J Clin Oncol 17(9):2639-2648

8. Romond EH, Perez EA, Bryant J et al (2005) Trastuzumab plus adjuvant chemotherapy for operable HER2-positive breast cancer. N Engl J Med 353(16):1673-1684

9. Gown AM, Goldstein LC, Barry TS et al (2008) High concordance between immunohistochemistry and fluorescence in situ hybridization testing for HER2 status in breast cancer requires a normalized IHC scoring system. Mod Pathol 21(10):1271

10. Jelovac D, Emens LA (2013) HER2-directed therapy for metastatic breast cancer. Oncol. 27(3):166

11. Olsen D, Jørgensen JT (2014) Companion diagnostics for targeted cancer drugs-clinical and regulatory aspects. Front Oncol. https ://doi.org/10.3389/fonc.2014.00105

12. Hui L, Geiersbach KB, Downs-Kelly E, Gulbahce HE (2016) RAI1 Alternate probe identifies additional breast cancer cases as amplified following equivocal HER2 fluorescence in situ hybridization testing: experience from a national reference laboratory. Arch Pathol Lab Med 141(2):274-278

13. Press MF, Sauter G, Buyse M et al (2016) HER2 gene amplification testing by fluorescent in situ hybridization (FISH): comparison of the ASCO-college of American Pathologists guidelines with FISH scores used for enrollment in breast cancer international research group clinical trials. J Clin Oncol 34(29):3518-3528

14. Ragazzi M, Bisagni A, Gasparini E et al (2017) Impact of 2013 ASCO/CAP guidelines on HER2 determination of invasive breast cancer: a single institution experience using frontline dual-color FISH. The Breast. 34:65-72

15. Zhang X, Bleiweiss I, Jaffer S, Nayak A (2017) The impact of 2013 updated ASCO/CAP HER2 guidelines on the diagnosis and management of invasive breast cancer: a single-center study of 1739 cases. Clin Breast Cancer. https://doi.org/10.1016/j. clbc.2017.03.012

16. Bethune GC, Veldhuijzen van Zanten D, MacIntosh RF et al (2015) Impact of the 2013 American society of clinical oncology/ college of American pathologists guideline recommendations for human epidermal growth factor receptor 2 (HER2) testing of invasive breast carcinoma: a focus on tumours assessed as 'equivocal' for HER2 gene amplification by fluorescence in situ hybridization. Histopathology 67(6):880-887

17. Pennacchia I, Carbone A, Di Cerbo A, Vecchio FM, Arena V (2015) 2013 ASCO/CAP updated guidelines for human epidermal growth factor receptor 2 testing: impact on routine practice. The Breast. 24(3):285-286

18. Panigrahi MK, Kumar D, Mehta A, Saikia KK (2017) Outcome of HER2 Testing by FISH applying ASCO/CAP 2007 and 2013 guideline in IHC equivocal group of breast cancer: experience at tertiary cancer care centre. South Asian J Cancer. 6(2):45

19. Long TH, Lawce H, Durum C et al (2015) The new equivocal: changes to HER2 FISH results when applying the 2013 ASCO/ CAP guidelines. Am J Clin Pathol 144(2):253-262

20. Wolff AC, Hammond MEH, Schwartz JN et al (2007) American Society of clinical oncology/college of American Pathologists guideline recommendations for human epidermal growth factor receptor 2 testing in breast cancer. Arch Pathol Lab Med 131(1):18-43

21. Tse CH, Hwang HC, Goldstein LC et al (2011) Determining true HER2 gene status in breast cancers with polysomy by using 
alternative chromosome 17 reference genes: implications for antiHER2 targeted therapy. J Clin Oncol 29(31):4168-4174

22. Varga Z, Tubbs RR, Wang Z et al (2012) Co-amplification of the HER2 gene and chromosome 17 centromere: a potential diagnostic pitfall in HER2 testing in breast cancer. Breast Cancer Res Treat 132(3):925-935

23. Hanna WM, Rüschoff J, Bilous M et al (2014) HER2 in situ hybridization in breast cancer: clinical implications of polysomy 17 and genetic heterogeneity. Mod Pathol 27(1):4

24. Liu Y, Ma L, Liu D et al (2014) Impact of polysomy 17 on HER2 testing of invasive breast cancer patients. Int J Clin Exp Pathol. 7(1): 163

25. Troxell ML, Bangs CD, Lawce HJ et al (2006) Evaluation of Her-2/neu status in carcinomas with amplified chromosome 17 centromere locus. Am J Clin Pathol 126(5):709-716

26. Sahlberg KK, Hongisto V, Edgren $\mathrm{H}$ et al (2013) The HER2 amplicon includes several genes required for the growth and survival of HER2 positive breast cancer cells. Mol Oncol. 7(3):392-401

27. Viale G (2009) Be precise! The need to consider the mechanisms for CEP17 copy number changes in breast cancer. J Pathol. 219(1):1-2

28. Wolff AC, Hammond MEH, Hicks DG et al (2013) Recommendations for human epidermal growth factor receptor 2 testing in breast cancer: American Society of clinical oncology/College of American Pathologists clinical practice guideline update. J Clin Oncol 31(31):3997-4013

29. Pinhel I, Hills M, Drury S et al (2012) ER and HER2 expression are positively correlated in HER2 non-overexpressing breast cancer. Breast Cancer Res 14(2):R46
30. Eswarachary V, Mohammed IG, Jayanna PK (2017) HER2/neu testing in 432 consecutive breast cancer cases using FISH and IHC-A comparative study. J Clin Diagn Res 11(4):EC01-EC05

31. Solomon JP, Dell'Aquila M, Fadare O, Hasteh F (2017) Her2/ neu status determination in breast cancer: a single institutional experience using a dual-testing approach with immunohistochemistry and fluorescence in situ hybridization. Am J Clin Pathol 147(4):432-437

32. Yaziji H, Goldstein LC, Barry TS et al (2004) HER-2 testing in breast cancer using parallel tissue-based methods. JAMA 291(16): 1972-1977

33. Dowsett M, Bartlett J, Ellis I et al (2003) Correlation between immunohistochemistry (HercepTest) and fluorescence in situ hybridization (FISH) for HER 2 in 426 breast carcinomas from 37 centres. J Pathol. 199(4):418-423

34. Owens MA, Horten BC, Da Silva MM (2004) HER2 amplification ratios by fluorescence in situ hybridization and correlation with immunohistochemistry in a cohort of 6556 breast cancer tissues. Clin Breast Cancer. 5(1):63-69

35. Beatty BG, Bryant R, Wang W et al (2004) HER-2/neu detection in fine-needle aspirates of breast cancer: fluorescence in situ hybridization and immunocytochemical analysis. Am J Clin Pathol 122(2):246-255

36. Pauletti G, Dandekar S, Rong H et al (2000) Assessment of methods for tissue-based detection of the HER-2/neu alteration in human breast cancer: a direct comparison of fluorescence in situ hybridization and immunohistochemistry. J Clin Oncol 18(21):3651-3664 